\title{
ESTUDO DOS REFLEXOS PRIMITIVOS EM PACIENTES RECÉM-NASCIDOS PRÉ-TERMO NORMAIS NO PRIMEIRO ANO DE VIDA
}

\author{
Lygia Olhweiler ${ }^{1}$, Alexandre Rodrigues da Silva², Newra Tellechea Rotta ${ }^{3}$
}

\begin{abstract}
RESUMO - Foi realizado estudo não controlado, de coorte histórica, com o objetivo de estabelecer marcos do desenvolvimento neurológico, definir o perfil clínico e epidemiológico de recém nascidos prematuros normais aos 3, 6, 9 e 12 meses de idade corrigida quanto à evolução dos reflexos primitivos. Resultados: Aos 3 meses de idade corrigida, todos os reflexos primitivos estavam presentes em diferentes percentuais. Aos 6 meses de idade corrigida, $100 \%$ das crianças apresentavam preensão plantar e 2,7\% apresentavam reflexo de Moro, apoio plantar e preensão palmar. A preensão plantar foi o único reflexo primitivo observado aos 9 e 12 meses de idade corrigida. Conclusão: Foi possível avaliar a ocorrência, assim como o desaparecimento dos reflexos primitivos de recém-nascidos prematuros normais. Estes resultados apontam para um atraso no desaparecimento dos reflexos primitivos mesmo com o uso da correção da idade.
\end{abstract}

PALAVRAS-CHAVE: desenvolvimento neuropsicomotor, prematuridade, recém-nascidos.

\section{Primitive reflex in premature healthy newborns during the first year}

ABSTRACT - A non-controlled, prognostic cohort study was performed with the aim of establishing markers of neurological development and defining a clinical and epidemiological profile of preterm newborns at $3,6,9$, and 12 months of gestation-corrected age in terms of primitive reflexes evolution. Results: At 3 months old of corrected age, all primitive reflexes were present. At 6 months old, all children showed plantar grasp and 2,7\% still showed Moro and palmar grasp. Plantar grasp was the unique primitive reflex found at 9 and 12 months of corrected age. Conclusion: It was possible to evaluate the occurence, as well as the disappearing of primitive reflexes in preterm newborns. The results show delay in the disappearing of primitive reflexes even with the use of corrected age.

KEY WORDS: psychomotor development, preterm newborn.

O desenvolvimento da criança do ponto de vista neuropsicossensorial e motor depende do processo de maturação do sistema nervoso central (SNC), principalmente no primeiro ano de vida ${ }^{1-6}$. Lefèvre ${ }^{7}$ e Diament ${ }^{8}$ demonstraram que o processo de maturação está relacionado com o grau de mielinização, arborização e formação de sinapses das células nervosas no SNC, que aos poucos vão inibindo as atividades reflexas primitivas, passando por uma fase de transição e, por último, assumindo o comando voluntário dessas atividades, que somente permanecerão em condições patológicas nos pacientes com lesão cerebral.

A maturação do SNC permite, além da inibição da atividade reflexa primitiva presente no recémnascido (RN) prematuro, o desenvolvimento das reações de retificação, de proteção e de equilíbrio (atividade reflexa postural), o desenvolvimento intelectual e das funções sensoriais de forma harmônica e integrada ${ }^{2,3,6}$.

O objetivo trabalho é verificar a presença e a evolução dos reflexos primitivos em crianças nascidas prematuras normais através do exame neurológico observando a idade corrigida ${ }^{1,8}$.

\section{MÉTODO}

A população estudada foi constituída de coorte de RN prematuros normais, acompanhados até 12 meses de idade corrigida, nascidos no Hospital de Clínicas de Porto Alegre (HCPA) no período de agosto de 1995 a novembro de 1998. As crianças foram acompanhadas no ambulatório de prematuros aos 3, 6, 9 e 12 meses de idade corrigida. Foram incluídos no estudo RN com

Unidade de Neurologia Infantil, Departamento de Pediatria do Hospital de Clínicas de Porto Alegre, Universidade Federal do Rio Grande do Sul, Porto Alegre RS, Brasil (UFRS): ${ }^{1}$ Neurologista Infantil, Doutora em Pediatria da UFRS; ${ }^{2}$ Médico Residente em Neurologia Infantil; ${ }^{3}$ Chefe da Unidade de Neurologia Infantil, Hospital de Clínicas de Porto Alegre.

Recebido 3 Maio 2004, recebido na forma final 20 Outubro 2004. Aceito 3 Dezembro 2004.

Dr. Alexandre Silva - Rua Abram Goldsztein 82 / Torre 1 / Ap 504 - 91450-155 Porto Alegre RS - Brasil. E-mail: alex164@ig.com.br 
idade gestacional de até 36 semanas e 6 dias, com $2000 \mathrm{~g}$ ou menos de peso no nascimento. A idade gestacional foi determinada pelo método de Ballard.

Foram excluídos RN que apresentaram uma ou mais das seguintes intercorrências: índices de Apgar $<7$ no $5^{\circ}$ minuto; hemorragia cerebral; crises convulsivas; alterações do estado de consciência; infecção do SNC; infecções congênitas; síndromes genéticas e intoxicações pré-natais; intercorrências clínicas capazes de interferir no desenvolvimento neurológico, tais como sepse grave (alteração da perfusão periférica, pressão arterial, distúrbio do equilíbrio ácido-básico e da coagulação); icterícia com necessidade de exsanguineotransfusão; ventilação mecânica por mais de 7 dias e apnéias com repercussões hemodinâmicas.

Foram excluídas, no decorrer do acompanhamento, as crianças que apresentaram intercorrências capazes de interferir na evolução do SNC, tais como convulsões, traumatismo cranioencefálico com sintomatologia neurológica, infecções do SNC, exame neurológico alterado, potenciais evocados auditivo e visual e ultra-sonografia cerebral alterados no período neonatal.

Entraram no estudo todos os RN nascidos no HC PA no período estudado que preencheram os critérios estabelecidos. Foram avaliados através da história, do exame neurológico e da determinação da idade gestacional até $05^{\circ}$ dia de vida. As crianças foram acompanhadas durante a permanência no berçário e encaminhadas para o ambulatório de prematuros para avaliação aos 3, 6, 9 e 12 meses de idade corrigida. Para o cálculo da idade corrigida, foi considerada idade gestacional de 40 semanas como de termo. As avaliações foram realizadas sempre pelo mesmo examinador.

$O$ presente estudo foi submetido à análise da Comissão Coordenadora do Curso de Pós- Graduação em Medicina-Pediatria da Universidade Federal do Rio Grande do Sul e da Comissão de Pesquisa e Ética em Saúde do Grupo de Pesquisa e Pós-Graduação do HCPA aprovado sob o $\mathrm{n}^{\circ} 95.075$.
Foi realizada análise estatística por meio dos softwares EPI-INFO versão 6.04 e SPSS. Em relação à avaliação neurológica, foram descritas freqüências e seus respectivos intervalos de confiança em $95 \%$.

\section{RESULTADOS}

Antecedentes gineco-obstétricos e características dos $R N$ - Todas as mães tiveram cuidados prénatais; $52,5 \%$ delas eram primíparas. Pré-eclâmpsia $(32,5 \%)$ e ruprema $(22,5 \%)$ foram as intercorrências mais freqüentes encontradas na história gestacional. Tipo de parto: $45 \%$ dos nascimentos ocorreram por parto vaginal e $55 \%$ por cesareana. A distribuição dos RN quanto à idade gestacional, avaliada pelo método de Ballard até o $5^{\circ}$ dia de vida, revelou que $25 \%$ deles apresentavam idade gestacional abaixo de 30 semanas, $48 \%$ se situaram entre 30 34 semanas e $27 \%$ tinham acima de 34 semanas de idade gestacional. Quanto ao peso no nascimento, 50,5\% dos RN tinham entre 1000 e $1500 \mathrm{~g}$, $20 \%$ pesavam menos de $1000 \mathrm{~g}$ e $29,5 \%$ tinham acima de $1500 \mathrm{~g}$. Dos $40 \mathrm{RN}$ prematuros incluídos no estudo, $75 \%$ foram classificados como adequados para a idade gestacional e $25 \%$ como pequenos para a idade gestacional na análise da adequação do peso em relação à idade gestacional. Em relação ao Apgar no $5^{\circ}$ minuto, 32,5\% dos RN tiveram Apgar 8 e $9,5 \%$ apresentaram Apgar 7 e $10 \%$ ficaram com Apgar 10. O tempo médio de permanência no hospital foi $44 \pm 3$ dias. Icterícia neonatal foi a intercorrência mais freqüente $(92,3 \%)$, seguida de sepse $(82,5 \%)$, apnéia da prematuridade $(56,4 \%)$, necessidade de CPAP $(42,5 \%)$ e de VM $(28,3 \%)$.

Durante o período de agosto de 1995 a novembro de 1998, ocorreram 695 nascimentos de crianças com idade gestacional até 36 semanas e 6 dias e de até $2000 \mathrm{~g}$ de peso, no HCPA. Foram selecionados 42

Tabela 1. Reflexos primitivos nos diferentes períodos.

\begin{tabular}{lcccccccc}
\hline Idade & \multicolumn{2}{c}{3 meses } & \multicolumn{2}{c}{6 meses } & \multicolumn{2}{c}{9 meses } & \multicolumn{2}{c}{12 meses } \\
Reflexos & $\%$ & IC & $\%$ & IC & $\%$ & IC & $\%$ & IC \\
\hline Suç̧ão & 97,1 & $85-100$ & 0 & $0-10$ & 0 & $0-9$ & 0 & $0-10$ \\
Moro & 77,1 & $60-90$ & 2,7 & $0-14$ & 0 & $0-9$ & 0 & $0-10$ \\
Apoio plantar & 54,3 & $37-71$ & 2,7 & $0-14$ & 0 & $0-9$ & 0 & $0-10$ \\
Marcha & 57,1 & $39-74$ & 0 & $0-10$ & 0 & $0-9$ & 0 & $0-10$ \\
Reptação & 48,6 & $31-66$ & 0 & $0-10$ & 0 & $0-9$ & 0 & $0-10$ \\
Preensão palmar & 91,4 & $77-98$ & 2,7 & $0-14$ & 0 & $0-9$ & 0 & $0-10$ \\
Preensão plantar & 100 & $99-100$ & 100 & $91-100$ & 97,4 & $87-100$ & 35,3 & $20-54$ \\
RCPF & 0 & $0-10$ & 0 & $0-10$ & 5,3 & $1-18$ & 66,7 & $48-92$ \\
Mão - boca & 51,4 & $34-69$ & 0 & $0-10$ & 0 & $0-9$ & 0 & $0-10$ \\
\hline
\end{tabular}

IC, intervalo de confiança; RCPF, reflexo cutâneo plantar flexor. 
RN prematuros que preencheram os critérios de inclusão, dos quais um apresentou no seguimento alterações no exame neurológico e um transferiuse para outro Estado, não continuando o acompanhamento. Os 40 prematuros foram acompanhados de forma sistematizada no ambulatório de desenvolvimento de prematuros do HCPA, com total aderência destes ao protocolo de pesquisa, sendo este o primeiro seguimento neurológico de prematuros no nosso meio. Dos 40 RN prematuros, $24(60 \%)$ eram do sexo feminino e 16 (40\%) do sexo masculino, todos de cor branca. Aos 3 meses de idade corrigida, todos os reflexos primitivos estavam presentes em diferentes percentuais. Aos 6 meses de idade corrigida, $100 \%$ das crianças apresentavam preensão plantar e $2,7 \%$ dos casos apresentavam reflexo de Moro, apoio plantar e preensão palmar. A preensão plantar foi o único reflexo primitivo observado aos 9 e 12 meses de idade corrigida (Tabela 1).

\section{DISCUSSÃO}

Os períodos precoces da vida intra-uterina, peri, pós-natal e no primeiro ano de vida têm merecido atenção dos pesquisadores, por serem capazes de trazer informações úteis para o entendimento de futuras alterações do desenvolvimento da criança9. Embora o desenvolvimento de cuidados intensivos permita a sobrevivência de prematuros cada vez menores, a incidência de patologias neurológicas que inviabilizem sobrevivência em condições adequadas não tem se modificado. A avaliação neurológica das crianças é peculiar, porque é necessário diferenciá-la nas diversas etapas do desenvolvimento, que incluem o RN prematuro, de termo, o lactente, a criança e o adolescente a $^{3,6-8,10-16}$.

Há um caráter evolutivo do exame neurológico do RN que faz com que seja necessária a divisão dos achados por idades chaves ${ }^{17,18}$. Vários estudos têm demonstrado a importância do uso da idade corrigida ao se estudar o desenvolvimento neuropsicomotor de prematuros ${ }^{19-23}$. As alterações mais freqüentes encontradas no exame neurológico nos estudos de acompanhamento de prematuros são paralisia cerebral e alterações visuais e auditi$\operatorname{vas}^{24,25}$. No tempo que durou esta pesquisa o exame neurológico permaneceu normal.

Os reflexos primitivos são reações automáticas desencadeadas por estímulos que impressionam diversos receptores e que compartilham, com o resto do processo evolutivo, as características dinâmicas da maturação infantil ${ }^{6}$. A pesquisa dos reflexos pri- mitivos constitui-se numa ferramenta útil para verificar a integridade do sistema nervoso de RN de termo ${ }^{14,26}$. Algumas manifestações reflexas primitivas desaparecem durante os 6 primeiros meses de vida, reaparecendo no $2^{\circ}$ semestre como atividade motora voluntária; outras devem desaparecer com a evolução normal do sistema nervoso e serão observadas somente em condições patológicas ${ }^{2,3,9}$.

Allen e Capute ${ }^{5}$ realizaram estudo de coorte com 47 prematuros a partir de 25 semanas de idade gestacional, pesquisando os reflexos primitivos semanalmente. Eles concluíram que os prematuros apresentavam o mesmo comportamento do RN de termo ao atingir 40 semanas de idade gestacional. Piper et al. ${ }^{26}$ compararam dois grupos de prematuros de baixo risco aos 4 meses de idade corrigida e cronológica, divididos conforme a idade gestacional em menores de 32 semanas e maiores ou iguais a 32 semanas. Os autores encontraram diferença estatisticamente significativa em relação aos reflexos primitivos, concluindo que eles sofrem influência da maturação biológica e das experiências ambientais.

Neste estudo, os reflexos primitivos ainda estavam presentes na maioria das crianças aos 3 meses de idade corrigida; aos 6 meses, reflexo de Moro, apoio plantar e preensão palmar foram observados em $2,7 \%$ da amostra, e preensão plantar em $100 \%$ dos casos. Este reflexo foi o único que se manteve até os 12 meses de idade corrigida (Tabela 1). Tais resultados concordam com os de autores que estudaram a evolução dos reflexos primitivos em $\mathrm{RN}$ de termo normais durante o primeiro ano de vida ${ }^{2,6,8,9}$.

Na presente pesquisa, foi possível observar a correlação entre o reflexo cutâneo-plantar e a preensão plantar. O reflexo cutâneo-plantar se tornou flexor ao mesmo tempo que ocorreu o desaparecimento da preensão plantar.

Os resultados desta pesquisa, quando comparados aos de Diament ${ }^{8}$, utilizado como referência, mostram diferenças em relação à sucção, à preensão dos dedos e artelhos e aos reflexos de Moro e marcha.

Estas diferenças apontam para o fato de que, apesar de comparáveis, os dois estudos incluindo 40 RN utilizaram metodologia diferente, uma vez que o estudo de Diament ${ }^{8}$ não acompanhou, no primeiro ano de vida, o mesmo grupo de RN, mas usou crianças diferentes em cada idade-chave. É possível que o acompanhamento do mesmo grupo em cada etapa tenha um resultado mais fidedigno. Deve ser considerado também que a observação de dois pesquisadores em épocas diferentes pode não 
ser totalmente superponível. O fato de que, quanto mais próximo dos 12 meses, menores foram as diferenças observadas concorda com a literatura ${ }^{14,18}$.

Em conclusão, foi possível avaliar a ocorrência, assim como o desaparecimento dos reflexos primitivos de pacientes prematuros. Estes resultados apontam para um atraso no desaparecimento dos reflexos primitivos mesmo com o uso da correção da idade.

\section{REFERÊNCIAS}

1. Saint-Anne Dargassies S. Le nouveau-né à terme, aspect neurologique. Biol Néo-nat 1962;4:174-200.

2. Paine RS, Brazelton TB, Donovan DE, Drorbaugh JE, Hubbell JP, Sears M. Evolution of postural reflexes in normal infants and in the presence of chronic brain syndromes. Neurology 1964;4:1036-1048.

3. Lefèvre AB. Exame neurológico da criança. In Tolosa, APM Canelas HM (eds) Propedêutica neurológica, 2.Ed. São Paulo: Sarvier, 1975.

4. Brunet $\mathrm{O}$, Lézine I. O desenvolvimento psicológico da primeira infância. Porto Alegre: Editora Artes Médicas, 1981.

5. Allen MC, Capute AJ. Tone and reflex development before term. Pediatrics 1990;85:393-399.

6. Coriat LF. Maturação psicomotora no primeiro ano de vida da criança, 3.Ed. São Paulo: Moraes, 1991.

7. Lefèvre AFB. Contribuição para a padronização do exame neurológico do recém-nascido normal. Tese Faculdade de Medicina da Universidade de São Paulo; 1950

8. Diament AJ. Contribuição para a sistematização do exame neurológico de crianças normais no primeiro ano de vida. Tese, Faculdade de Medicina da Universidade de São Paulo São Paulo; 1967.

9. Pedroso FS, Rotta NT. From the foot-mouth reflex to the hand- mouth reflex: a continuum of responses to appendicular compression. Arq Neuropsiquiatr 1997;55:186-192.

10. Koupernik C. Desarollo psicomotor de la primeira infancia, 5.Ed. Barcelona: Editorial Luis Miracle AS, 1968.
11. Rotta NT. Desenvolvimento psicomotor. Pesq Méd 1973; 9:617-628.

12. Rotta NT. Problemas neurológicos do recém-nascido: o exame neurológico. In Miura E (ed). Neonatologia: princípios e prática. Porto Alegre: Artes Médicas, 1991.

13. Escobedo MB. Follow-up of prematurely born infants. Clin Obstet Gynecol 1988;31:54-77.

14. Saint-Anne Dargassies S. Desarollo neurológico del recien-nascido de termino y prematuro. Buenos Aires: Ed Médica Panamericana, 1977.

15. Amiel-Tison C, Grenier A. Normal development during the first year of life. In: Amiel-Tison C, Grenier A. (eds) New York: Masson, 1983.

16. Illingworth RS. The development of the infant and young child. normal and abnormal, 9.Ed. Edinburgh: Churchill Livingstone, 1987.

17. Saint-Anne Dargassies S. Méthode d'examen neurologique du nouveauné. Études Néo-Natales 1954;2:101-123.

18. Saint-Anne Dargassies S. Normality and normalization as seen in a longterm neurological follow-up of 286 truly premature infants. Neuropaediatri 1979;10:226-244.

19. Ungerer JA, Sigman M. Developmental lags in preterm infants from one to three years of age. Child Dev 1983;54:1217-1228.

20. Matilainen $R$. The value of correction for age in the assessment of prematurity born children. Early Hum Dev 1987;15:257-264.

21. Stjernqvist K, Svenningen NW. Extremely low birth weight infants less than 901g: development and behaviour after 4 years of life. Acta Paediatr 1995;84:500-506.

22. Mandich M, Simons CJ, Ritchie S, Schmidt D, Mullett M. Motor development, infantile reactions and postural responses of preterm, at-risk infants. Dev Med Child Neurol 1994;36:397-405.

23. Stewart AL, Reynolds EOR, Lipscomb AP. Outcome for infants of very low birthweight: survey of world literature. Lancet 1981;9:1038-1041.

24. Allen MC, Alexander GR. Gross motor milestones in preterm infants: correction for degree of prematurity. J Pediatr. 1990;116:955-959.

25. Roth S, Wyatt J, Baudin J, et al. Neurodevelopmental status at 1 year predicts neuropsychiatric outcome at $14-15$ years of age in very preterm infants. Early Hum Dev 2001;65:81-89.

26. Piper MC, Darrah J, Byrne P. Impact of gestational age on preterm motor development at 4 months chronological and adjusted ages. Child Care Health Dev 1989;15:105-115. 